\title{
Dimensional reduction of BPS attractors in AdS gauged supergravities
}

\author{
Kiril Hristov \\ Dipartimento di Fisica, Università di Milano-Bicocca, \\ Piazza della Scienza 3, I-20126 Milano, Italy \\ INFN, sezione di Milano-Bicocca, \\ Piazza della Scienza 3, I-20126 Milano, Italy \\ E-mail: kiril.hristov@unimib.it
}

ABSTRACT: We relate across dimensions BPS attractors of black strings and black holes of various topology in gauged supergravities with nontrivial scalar potential. The attractors are of the form $\mathrm{AdS}_{2,3} \times \Sigma^{2,3}$ in 4, 5, and 6 dimensions, and can be generalized to some higher dimensional analogs. Even though the attractor geometries admit standard Kaluza-Klein and Scherk-Schwarz reductions, their asymptotic AdS spaces in general do not. The resulting lower dimensional objects are black holes with runaway asymptotics in supergravity theories with no maximally symmetric vacua. Such classes of solutions are already known to exist in literature, and results here suggest an interpretation in terms of their higher-dimensional origin that often has a full string theory embedding. In a particular relevant example, the relation between 5d Benini-Bobev black strings [1, 2] and a class of 4d Cacciatori-Klemm black holes [3] is worked out in full detail, providing a type IIB and dual field theory description of the latter solutions. As a consistency check, the Cardy formula for the field theory is shown to match the Bekenstein-Hawking entropy for horizon topology of any genus.

KeYwords: Black Holes in String Theory, Supergravity Models, AdS-CFT Correspondence

ARXIV EPRINT: 1409.8504 


\section{Contents}

1 Introduction 1

2 Dimensional reduction of gauged theories 3

3 5d to 4 d: black string $\rightarrow$ black hole $\quad 4$

3.1 Benini-Bobev black strings 4

$\begin{array}{ll}3.2 & \text { Caciatori-Klemm black holes }\end{array}$

$\begin{array}{lll}3.3 & \text { Dimensional reduction } & 7\end{array}$

3.4 Matching asymptotics 8

$\begin{array}{ll}3.5 & \text { Superalgebras and Killing spinors }\end{array}$

3.6 Dual field theory and Cardy formula 9

$\begin{array}{ll}3.7 & \text { Extensions with extra charges } \\ & 10\end{array}$

4 6d to 5d: black string $\rightarrow$ black hole/string $\quad 10$

$\begin{array}{lll}4.1 & \text { Reduction along } \mathrm{AdS}_{3} & 11\end{array}$

$\begin{array}{lll}4.2 & \text { Reduction along } \mathrm{S}^{3} & 11\end{array}$

4.3 Reduction along both $\mathrm{AdS}_{3}$ and $\mathrm{S}^{3} \quad 12$

\section{Introduction}

The topic of dimensional reduction between supergravity theories and their vacua has been a very fruitful area of research for the development of string theory [4-12]. The relation between supersymmetric black (st)rings and black holes (even if not made fully explicit at the time) in ungauged supergravities in $6 \mathrm{~d} / 5 \mathrm{~d}$ and $5 \mathrm{~d} / 4 \mathrm{~d}$ was crucial for the microscopic understanding of black hole entropy $[13,14]$ and has therefore given us a tool to look into the quantum regime of black hole physics. Following this train of thought, here and in a companion paper [15] we explore similar relations between BPS black objects in 4, 5, and 6 dimensions, this time in gauged supergravity. In particular here we look at the dimensional reduction for gauged theories with a nontrivial scalar potential, starting from asymptotically $\mathrm{AdS}_{5,6}$ black hole solutions and using standard Kaluza-Klein and ScherkSchwarz reduction ansätze.

The question of direct dimensional reduction of full black hole spacetimes from 6 to 5 or from 5 to 4 dimensions is slightly more subtle in the presence of scalar potential than in the absense of one. It is not possible geometrically to construct a relation $\mathrm{AdS}_{6} \rightarrow \mathrm{AdS}_{5} \rightarrow \mathrm{AdS}_{4}$ by declaring that one spatial dimension becomes compact and small, as in the case $\operatorname{Mink}_{6} \rightarrow$ Mink $_{5} \rightarrow$ Mink$_{4}$. However, even in the presence of scalar potential one can still relate the near-horizon geometry $\mathrm{AdS}_{3} \times \mathrm{S}^{3}$ (or a more general 3-surface $\Sigma^{3}$ ) of the 6 dimensional black string to the lower-dimensional near-horizon geometries $\mathrm{AdS}_{2} \times \mathrm{S}^{3}$ 
and $\mathrm{AdS}_{3} \times \mathrm{S}^{2}$ of the $5 \mathrm{~d}$ black holes and strings and eventually to the $4 \mathrm{~d}$ black hole attractor $\mathrm{AdS}_{2} \times \mathrm{S}^{2}$ (as already done in [16] in ungauged supergravity). The near-horizon geometries of the lower-dimensional solutions cannot be anymore connected by an RG flow to asymptotic AdS flows as the original ones, but rather to curved domain walls [3].

This relation between attractors is rather general and can be applied to a variety of already known or yet to be found solutions in gauged supergravities of various dimensions. It can be used as an additional tool to classify supergravity solutions in theories with runaway behavior that might on first sight seem ill defined. When lifted to one extra dimension these solutions become perfectly good and should therefore be taken seriously, which is the main message of this paper.

The explicit example worked out in detail here relates two well-known classes of black objects. If we take a black string in 5 d, i.e. a spacetime interpolating between $\operatorname{AdS}_{3} \times \Sigma^{2}$ and $\mathrm{AdS}_{5}$, the $4 \mathrm{~d}$ result after dimensional reduction along the circle in $\mathrm{AdS}_{3}$ looks like a solution interpolating between $\mathrm{AdS}_{2} \times \Sigma^{2}$ and a non-maximally symmetric runaway vacuum. Such classes of solutions are already known [3] and it is interesting to observe that such a dimensional reduction can preserve the full amount of supersymmetry. It turns out that the BPS black strings summarized in $[1,2]$ upon dimensional reduction give a class of BPS black hole solutions with curved domain wall asymptotics found in [3] for the $4 \mathrm{~d}$ $N=2$ supergravity prepotential

$$
F=\frac{X^{1} X^{2} X^{3}}{X^{0}}
$$

This suggests a more transparent string theory interpretation of the $4 \mathrm{~d}$ solutions as asymptotic $\mathrm{AdS}_{5} \times \mathrm{S}^{5}$ states in type IIB string thery with well-known field theory dual. One can even find much about the superconformal field theory living on the horizon of these solutions from RG-flow techniques developed in $[1,2]$. In this way the dimensional reduction allows us to transform a somewhat obscure $4 \mathrm{~d}$ object into a full string theory background with a well-understood microscopic description. We stress that via the dimensional reduction the class of Cacciatori-Klemm (CK) solutions with the above prepotential now have the interpretation of wrapped D3 branes. These are different from the probably better known class of CK solutions embeddable in 11d supergravity that have the interpretation of wrapped M2 branes, found in an $N=2$ supergravity theory with prepotential ${ }^{1}$

$$
F=-2 i \sqrt{X^{0} X^{1} X^{2} X^{3}} .
$$

The outline of the paper is as follows. In section 2 we discuss in more detail the general features of the reduction on a circle of theories with bare cosmological constant or scalar potential. We then go to the specific case of reduction between 5d black string and $4 \mathrm{~d}$ black hole attractors in section 3. This is done in full detail, discussing various aspects such as symmetry algebras and dual field theories. The explicit reduction further reveals a possible generalization of the known black string near-horizon geometries. We then move to the relation between $6 \mathrm{~d}$ and $5 \mathrm{~d}$ attractors in section 4 , which is sketched

\footnotetext{
${ }^{1}$ Here we make a distinction between two supergravity theories with prepotentials that are dual to each other in the ungauged case. However in both theories we allow only for electric gauging of the R-symmetry, therefore we break the duality and truly find two physically distinct models.
} 
more briefly with an emphasis on the new features such as the choice of compactification directions. After this point further generalizations to higher dimensions should be clear to the reader, examples of which can be reductions on end points of holographic RG flows a la Maldacena-Nunez [17] that can be found in $[1,2,18]$ and references therein.

\section{Dimensional reduction of gauged theories}

It is instructive to see in a simple example why the dimensional reduction on a circle of a theory of gauged supergravity leads to a lower dimensional supergravity theory with no maximally symmetric vacua. ${ }^{2}$ In particular, the lower dimensional theory cannot have an $\mathrm{AdS}_{d}$ vacuum if the original one had an $\mathrm{AdS}_{d+1}$ vacuum (for $d>2$ ). This is clear from geometric point of view since $\mathrm{AdS}_{d+1}$ is not an $\mathrm{S}^{1}$ fibre over $\mathrm{AdS}_{d}$, except when $d=2$. The special case of reduction from $\mathrm{AdS}_{3}$ to $\mathrm{AdS}_{2}$ (and the analogous one from $\mathrm{S}^{3}$ to $\mathrm{S}^{2}$ ) is exactly the reason why the dimensional reduction between near-horizon geometries of black objects of various dimensions works in first place.

Let us consider the simplest gravitational theory in $(d+1)$ dimensions with a cosmological constant, such that it has an $\mathrm{AdS}_{d+1}$ vacuum solution:

$$
S_{d+1}=\int \mathrm{d} x^{d+1} \sqrt{g_{d+1}}\left(R_{d+1}+\Lambda\right) .
$$

We can reduce to $d$-dimensions with the most-general Kaluza-Klein (KK) ansatz,

$$
\mathrm{d} s_{d+1}^{2}=e^{-2 \alpha \phi} \mathrm{d} s_{d}^{2}+e^{2 \beta \phi}\left(\mathrm{d} x_{d+1}+A_{m} \mathrm{~d} x^{m}\right)^{2},
$$

where $\phi$ and $A_{m}$ are the KK scalar and vector, respectively. Under the above decomposition we have that $\sqrt{g_{d+1}}=e^{(\beta-d \alpha) \phi} \sqrt{g_{d}}$. Since we would like to remain for simplicity in the Einstein frame in the lower dimensional theory ${ }^{3}$ we require that $\beta=(d-2) \alpha$. We therefore find that the lower dimensional action contains the following terms:

$$
S_{d}=\int \mathrm{d} x^{d} \sqrt{g_{d}}\left(R_{d}+\Lambda e^{-2 \alpha \phi}+\ldots\right)
$$

where the extra terms '...' are the kinetic terms for the KK scalar and vector, depending on the particular value of the parameter $\alpha$. One can see that the lower dimensional theory has a scalar potential that originates from the cosmological constant in (2.1),

$$
V=\Lambda e^{-2 \alpha \phi},
$$

which has no critical points. In fact the potential has a runaway behavior with a global extremum at $\phi \rightarrow \infty$, which is the decompactification limit. The gravitational ground state of the lower dimensional theory is in a sense trying to come back to its higher dimensional $\mathrm{AdS}_{d+1}$ origin. If one is only given the lower dimensional lagrangian (2.3) the conclusion would be that there is no ground state, and all solutions of the theory asymptote to a sort

\footnotetext{
${ }^{2}$ Note that there exist some very special examples of the opposite, see [19] for a $6 \mathrm{~d}$ theory admitting an $\mathrm{AdS}_{5} \times \mathrm{S}^{1}$ vacuum.

${ }^{3}$ There is no loss of generality in this choice, but the main argument here is easier to see this way.
} 
of curved domain wall (see more comments in section 3.3 of [3]). This feature is completely general and one can easily substitute the plain cosmological constant $\Lambda$ in (2.1) with a more complicated scalar potential that generically appears in supergravity theories. The appearance of the extra KK scalar from the metric reduction always means that the lowerdimensional scalar potential has no local extrema in the extra scalar direction, as we see in the folowing sections.

\section{$3 \quad 5$ d to 4 d: black string $\rightarrow$ black hole}

In order to show our main point and connect two classes of known solutions in 4 and 5 dimensional supergravities, ${ }^{4}$ we start from the Benini-Bobev (BB) black string solutions $[1,2]$. They are quarter-BPS numerical solutions interpolating between $\operatorname{AdS}_{3} \times \Sigma^{2}$ and asymptotically locally $\mathrm{AdS}_{5}$ foliated in $\mathbb{R} \times S^{1} \times \Sigma^{2}$ coordinates $\left(\Sigma^{2}\right.$ can be any compact Riemann surface). We already discussed above that one cannot expect a proper reduction to 4 dimensions of the full flow, therefore we concentrate in particular on the near-horizon reduction from $\mathrm{AdS}_{3} \times \Sigma^{2}$ down to $\mathrm{AdS}_{2} \times \Sigma^{2}$. Once this is done we can show that the resulting 4 dimensional geometry is still supersymmetric ${ }^{5}$ and has an asymptotic flow to a runaway vacuum, exactly as expected. This suggests that from a full 10-dimensional perspective one should rather think of the two solutions as being the same, the difference arising only when the extra dimensions are forgotten.

\subsection{Benini-Bobev black strings}

We consider $N=2 D=5$ Fayet-Iliopoulos (FI) gauged supergravity with two vector multiplets (the so-called STU model), which is a truncation of the maximal $N=8$ supergravity arising from the compactification of type IIB supergravity on $\mathrm{S}^{5}$. The bosonic fields are the metric $g_{\mu \nu}, 2$ real scalars $\phi^{1,2}$, and three abelian gauge fields $A_{\mu}^{1,2,3}$. The bosonic lagrangian in standard conventions [23] is given by

$$
\begin{aligned}
e^{-1} \mathcal{L}= & \frac{1}{2} R+g^{2} V-\frac{1}{4} G_{I J} F_{\mu \nu}{ }^{I} F^{\mu \nu J}-\frac{1}{2} \mathcal{G}_{i j} \partial_{\mu} \phi^{i} \partial^{\mu} \phi^{j} \\
& +\frac{e^{-1}}{48} \epsilon^{\mu \nu \rho \sigma \lambda} C_{I J K} F_{\mu \nu}^{I} F_{\rho \sigma}^{J} A_{\lambda}^{K},
\end{aligned}
$$

with a gauge coupling constant $g$ and a scalar potential given by

$$
V(X)=V_{I} V_{J}\left(6 X^{I} X^{J}-\frac{9}{2} \mathcal{G}^{i j} \partial_{i} X^{I} \partial_{j} X^{J}\right)
$$

where $X^{I}$ represent the real scalar fields and satisfy the condition $\mathcal{V}=\frac{1}{6} C_{I J K} X^{I} X^{J} X^{K}=$ 1. In the gauged STU model we further have $V_{1}=V_{2}=V_{3}=\frac{1}{3}$ and $C_{123}=1$ and its

\footnotetext{
${ }^{4}$ The relation between $4 \mathrm{~d}$ and $5 \mathrm{~d}$ flow equations for extremal black branes in gauged supergravity was already noted in [20]. Here we take a different approach and relate already known BPS solutions, rather than the underlying equations.

${ }^{5}$ Note that there is another possible supersymmetric reduction from $\mathrm{AdS}_{3} \times \Sigma^{2}$ down to $\mathrm{AdS}_{3}$ in $3 \mathrm{~d}$ gauged supergravity $[21,22]$. It provides further understanding of the gravitational counterpart of c-extremization.
} 
permutations as only nonvanishing components. The physical quantities in (3.1) can be expressed in terms of the homogeneous cubic polynomial $\mathcal{V}$. We also have the relations

$$
\begin{aligned}
G_{I J} & =-\left.\frac{1}{2} \partial_{I} \partial_{J} \log \mathcal{V}\right|_{\mathcal{V}=1}, \\
\mathcal{G}_{i j} & =\left.\partial_{i} X^{I} \partial_{j} X^{J} G_{I J}\right|_{\mathcal{V}=1},
\end{aligned}
$$

where $\partial_{i}$ and $\partial_{I}$ refer, respectively, to a partial derivative with respect to the scalar field $\phi^{i}$ and $X^{I}=X^{I}\left(\phi^{i}\right)$.

Note that the only difference between gauged and ungauged supergravity at the level of the presented bosonic lagrangian is the appearance of the scalar potential (3.2).

The near-horizon solutions in $[1,2]$, that summarize the earlier work of [24-28], are given by

$$
\begin{array}{rlrl}
\mathrm{d} s^{2} & =R_{A d S_{3}}^{2} \mathrm{~d} s_{A d S_{3}}^{2}+R_{\Sigma}^{2} \mathrm{~d} \sigma_{\Sigma}^{2}, & & \\
F^{I} & =-a_{I} V o l_{\Sigma}, & & \\
X^{1} & =e^{-\frac{\phi^{1}}{\sqrt{6}}-\frac{\phi^{2}}{\sqrt{2}}}, & X^{2}=e^{-\frac{\phi^{1}}{\sqrt{6}}+\frac{\phi^{2}}{\sqrt{2}}}, \quad X^{3}=e^{\frac{2 \phi^{1}}{\sqrt{6}}} .
\end{array}
$$

The solution is fully determined by the magnetic charges $a_{I}$ that satisfy the constraint

$$
a_{1}+a_{2}+a_{3}=-\kappa,
$$

with $\kappa=+1,-1$, or 0 depending on the curvature of the Riemann surface $\Sigma$ being positive, negative or zero. We have

$$
\begin{aligned}
e^{\sqrt{6} \phi^{1}} & =\frac{a_{3}^{2}\left(a_{1}+a_{2}-a_{3}\right)^{2}}{a_{1} a_{2}\left(-a_{1}+a_{2}+a_{3}\right)\left(a_{1}-a_{2}+a_{3}\right)}, & e^{\sqrt{2} \phi^{2}} & =\frac{a_{2}\left(a_{1}-a_{2}+a_{3}\right)}{a_{1}\left(-a_{1}+a_{2}+a_{3}\right)}, \\
R_{A d S_{3}}^{3} & =\frac{8 a_{1} a_{2} a_{3} \Pi}{\Theta^{3}}, & R_{\Sigma}^{6} & =\frac{a_{1}^{2} a_{2}^{2} a_{3}^{2}}{\Pi}
\end{aligned}
$$

with

$\Pi=\left(a_{1}+a_{2}-a_{3}\right)\left(a_{1}-a_{2}+a_{3}\right)\left(-a_{1}+a_{2}+a_{3}\right), \quad \Theta=2\left(a_{1} a_{2}+a_{1} a_{3}+a_{2} a_{3}\right)-\left(a_{1}^{2}+a_{2}^{2}+a_{3}^{2}\right)$.

We therefore find

$$
X^{1}=\frac{a_{1}\left(-a_{1}+a_{2}+a_{3}\right)}{\left(a_{1} a_{2} a_{3} \Pi\right)^{1 / 3}}, \quad X^{2}=\frac{a_{2}\left(a_{1}-a_{2}+a_{3}\right)}{\left(a_{1} a_{2} a_{3} \Pi\right)^{1 / 3}}, \quad X^{3}=\frac{a_{3}\left(a_{1}+a_{2}-a_{3}\right)}{\left(a_{1} a_{2} a_{3} \Pi\right)^{1 / 3}} .
$$

We can choose to write the metric of $\mathrm{AdS}_{3}$ locally in the extremal BTZ form

$$
\mathrm{d} s_{A d S_{3}}^{2}=\frac{1}{4}\left(-r^{2} \mathrm{~d} t^{2}+\frac{\mathrm{d} r^{2}}{r^{2}}\right)+\rho_{+}\left(\mathrm{d} y+\left(-\frac{1}{4}+\frac{r}{2 \rho_{+}}\right) \mathrm{d} t\right)^{2},
$$

with an arbitrary constant $\rho_{+}$that corresponds to the horizon radius of the extremal BTZ black hole such that upon reduction we have a more general solution with an arbitrary Kaluza-Klein electric charge, as done standardly in literature. Strictly speaking, the global properties of this metric are different than those of pure $\mathrm{AdS}_{3}$. However locally the metrics are the same, therefore the equations of motion and BPS variations are not sensitive under this change in the metric. 


\subsection{Caciatori-Klemm black holes}

Now we consider the analogous STU model, but in $N=2 D=4$ FI gauged supergravity with three vector multiplets. The bosonic fields are the metric $g_{\mu \nu}, 3$ complex scalars $z^{1,2,3}$, and four abelian gauge fields $A_{\mu}^{0,1,2,3}$. In standard $N=2$ conventions (see [29]), the lagrangian and susy variations are given in terms of symplectically covariant vectors such as to make the duality group manifest. The lagrangian is further uniquely defined by specifying the FI parameters $\xi_{1}=\xi_{2}=\xi_{3}=1$ and arbitrary $\xi_{0}$, and prepotential

$$
F=\frac{X^{1} X^{2} X^{3}}{X^{0}}
$$

where the $X^{\Lambda}\left(z^{i}\right)$ are the holomorphic sections of special geometry. With this choice of prepotential and FI parameters, the scalar potential is independent of $\xi_{0}$. For vanishing real part of the complex scalars, one finds the potential

$$
V(z) \sim \frac{1}{\operatorname{Im} z^{1}}+\frac{1}{\operatorname{Im} z^{2}}+\frac{1}{\operatorname{Im} z^{3}}
$$

which has no extrema and a typical runaway behavior exactly as explained in the previous section.

The Cacciatori-Klemm solutions and their generalizations $[3,30-35]$ in this theory can be found from the following equations ${ }^{6}$

$$
\begin{aligned}
\beta^{1}\left(-\beta^{1}+\beta^{2}+\beta^{3}\right) & =p^{1}, \\
\beta^{2}\left(\beta^{1}-\beta^{2}+\beta^{3}\right) & =p^{2}, \\
\beta^{3}\left(\beta^{1}+\beta^{2}-\beta^{3}\right) & =p^{3}, \\
\beta_{0}\left(\beta^{1}+\beta^{2}+\beta^{3}\right) & =q_{0},
\end{aligned}
$$

where the full solution for the metric and scalars is given by constants $\beta_{0}, \beta^{1,2,3}$ as in [34] and $q_{0}$ and $p^{1,2,3}$ are non-vanishing electric and magnetic charges carried by the gauge fields. We can already jump ahead of the dimensional reduction and adopt the notation where $p^{1} \equiv a_{1}, p^{2} \equiv a_{2}, p^{3} \equiv a_{3}$. Supersymmetry again imposes (3.7). The solution of the above equations is then

$$
\beta_{0}=q_{0} \frac{\sqrt{\Pi}}{\Theta}, \quad \beta^{1}=\frac{a_{1}\left(-a_{1}+a_{2}+a_{3}\right)}{\sqrt{\Pi}}, \quad \ldots
$$

with cyclic permutations of the indices for $\beta^{2,3}$. Two other useful identities are

$$
\beta^{1}+\beta^{2}+\beta^{3}=\frac{\Theta}{\sqrt{\Pi}}, \quad \beta^{1} \beta^{2} \beta^{3}=\frac{a_{1} a_{2} a_{3}}{\sqrt{\Pi}} .
$$

We can now in principle write down the full solution that flows between a near-horizon geometry and a runaway vacuum at infinity, called a curved domain wall in [3]. For the purposes of the explicit dimensional reduction we only concentrate on the horizon, since the

\footnotetext{
${ }^{6}$ Here we only look for axion-free solutions and use the conventions in [34]. Their generalizations with axions relate also to more general $5 \mathrm{~d}$ solutions of black strings with additional electric charges, see section 3.7.
} 
asymptotic geometry is already of the expected type that suggests its higher dimensional origin as explained in section 2. One finds the standard $\mathrm{AdS}_{2} \times \Sigma^{2}$ metric with different radii and scalars given by

$$
\begin{array}{rlrl}
\tilde{R}_{A d S_{2}}^{2} & =2 \frac{\sqrt{a_{1} a_{2} a_{3} q_{0}} \Pi}{\Theta^{5 / 2}}, & \tilde{R}_{\Sigma}^{2}=2 \frac{\sqrt{a_{1} a_{2} a_{3} q_{0}}}{\sqrt{\Theta}}, \\
\operatorname{Im} z^{1}=\frac{\sqrt{q_{0}}}{\sqrt{a_{1} a_{2} a_{3} \Theta}} a_{1}\left(-a_{1}+a_{2}+a_{3}\right), & \ldots
\end{array}
$$

with cyclic permutation for $z^{2,3}$.

Furthermore, observe that non-vanishing $q_{0}$ means that there is a nonvanishing electric component of the gauge field $A^{0}=\tilde{q}^{0} r \mathrm{~d} t$ with

$$
\tilde{q}^{0}=-\frac{\tilde{R}_{A d S_{2}}}{2 \tilde{R}_{\Sigma}^{2}} \mathcal{I}^{-1,00} q_{0}=\sqrt{\frac{a_{1} a_{2} a_{3}}{4 q_{0} \Theta}},
$$

where $\mathcal{I}^{-1}$ is the inverse of the imaginary part of the period matrix of special geometry (see again [29] for more details),

$$
\mathcal{I}^{-1,00}=-\frac{1}{\operatorname{Im}\left(z^{1} z^{2} z^{3}\right)} .
$$

\subsection{Dimensional reduction}

We now follow the standard rules of dimensional reduction from $5 \mathrm{~d}$ to $4 \mathrm{~d}$ supergravity, derived in $[8-10,12]$. Note that we already used the prepotential $(3.12)$ in $4 \mathrm{~d}$ that one finds from reducing the $5 \mathrm{~d}$ theory via the formula

$$
F=\frac{1}{6} \frac{C_{I J K} X^{I} X^{J} X^{K}}{X^{0}}
$$

together with the FI terms $\xi_{1}=\xi_{2}=\xi_{3}$. We did not specify $\xi_{0}$ as it can be left arbitrary also during the reduction - it is zero for a Kaluza-Klein reduction, or arbitrary non-zero constant for a more general Scherk-Schwarz reduction (see more in $[15]$ or $[6,7,11]$ ). It is a further consistency check to see that the reduction goes through independent of the value of $\xi_{0}$.

The rules for reducing the bosonic fields from 5 to 4 dimensions are the following:

$$
\begin{aligned}
\mathrm{d} s_{5}^{2} & =e^{2 \phi} \mathrm{d} s_{4}^{2}+e^{-4 \phi}\left(\mathrm{d} \gamma-A_{4}^{0}\right)^{2}, \\
A_{5}^{I} & =A_{4}^{I}+\operatorname{Re} z^{I}\left(\mathrm{~d} \gamma-A_{4}^{0}\right), \\
X_{5}^{I} & =2 e^{2 \phi} \operatorname{Im} z^{I},
\end{aligned}
$$

where the $4 \mathrm{~d}$ fields are already in the standard $4 \mathrm{~d} N=2$ conventions. Reducing the $5 \mathrm{~d}$ solution along $\gamma$ and identifying $e^{-2 \phi}=\rho_{+} R_{A d S_{3}}$, we end up with a $4 \mathrm{~d}$ solution of the form

$$
\begin{aligned}
\mathrm{d} s^{2} & =\frac{\rho_{+} R_{A d S_{3}}^{3}}{4} \mathrm{~d} s_{A d S_{2}}^{2}+R_{\Sigma}^{2} \rho_{+} R_{A d S_{3}} \mathrm{~d} \sigma_{\Sigma}^{2} \\
F^{0} & =\frac{1}{2 \rho_{+}} \operatorname{Vol}_{A d S_{2}}, \quad F^{I}=-a_{I} V o l_{\Sigma}
\end{aligned}
$$




$$
\operatorname{Im} z^{I}=\frac{\rho_{+} R_{A d S_{3}}}{2} X^{I}
$$

Therefore we expect an exact match between the following quantities upon the $5 \mathrm{~d}-4 \mathrm{~d}$ reduction:

$$
\tilde{R}_{A d S_{2}}^{2}=\frac{\rho_{+} R_{A d S_{3}}^{3}}{4}, \quad \tilde{R}_{\Sigma}^{2}=R_{\Sigma}^{2} \rho_{+} R_{A d S_{3}},
$$

together with the match of the scalars $z^{I}$ and field strengths. We have one arbitrary constant $\rho_{+}$that needs to reproduce correctly all these quantities and it turns out everything agrees exactly upon the identification

$$
\rho_{+}=\sqrt{\frac{q_{0} \Theta}{a_{1} a_{2} a_{3}}} .
$$

This concludes the proof that the near-horizon geometries of the BB solutions reduce to the near-horizon geometries of the CK solutions for the prepotential (3.12). More generally, this suggests that the full $4 \mathrm{~d}$ flow of the CK solutions between horizon and a curved domain wall is to be seen from a higher dimensional perspective as a black string in $\mathrm{AdS}_{5}$. In other words, the CK solutions of the $4 \mathrm{~d}$ gauged supergravity defined by the prepotential (3.12) are to be identified with the BB solutions and thus embedded in an asymptotically $\mathrm{AdS}_{5} \times \mathrm{S}^{5}$ background of type IIB string theory.

\subsection{Matching asymptotics}

To provide further evidence for the statement that the full CK solutions lift to the BB solutions in $5 \mathrm{~d}$, we can show how the asymptotic geometries match exactly. It would of course be desirable to show the full lift from the rules (3.20)-(3.22), but this is practically not possible since the BB solutions are known only numerically. One can instead hope in this way to discover the analytic form of the BB black strings in $5 \mathrm{~d}$, but this is not immediately obvious as it involves the construction of the additional function $e^{2 \phi}$ and we leave it for future investigations.

Concentrating only at the asymptotics, we find that the CK solutions we are looking at have the following runaway behavior for the metric and scalars at infinity (cf. section 3.3 of $[3])$ :

$$
\mathrm{d} s_{4, y \rightarrow \infty}^{2}=y^{3 / 2}\left(-\mathrm{d} t^{2}+\mathrm{d} \sigma_{\Sigma}^{2}\right)+y^{-3 / 2} \mathrm{~d} y^{2}, \quad \operatorname{Im} z_{y \rightarrow \infty}^{i} \sim y^{1 / 2} .
$$

Upon the coordinate change $r=y^{1 / 2}$, we find

$$
\mathrm{d} s_{4, r \rightarrow \infty}^{2}=r^{3}\left(-\mathrm{d} t^{2}+\mathrm{d} \sigma_{\Sigma}^{2}\right)+r^{-1} \mathrm{~d} r^{2}, \quad \operatorname{Im} z_{r \rightarrow \infty}^{i} \sim r,
$$

which can easily be uplifted to $\mathrm{AdS}_{5}$ with constant scalars via (3.20)-(3.22) upon identifying $e_{r \rightarrow \infty}^{2 \phi}=r^{-1}$,

$$
\mathrm{d} s_{5, r \rightarrow \infty}^{2}=r^{2}\left(-\mathrm{d} t^{2}+\mathrm{d} \sigma_{\Sigma}^{2}\right)+r^{-2} \mathrm{~d} r^{2}+r^{2} \mathrm{~d} \gamma^{2}, \quad X_{r \rightarrow \infty}^{I} \sim \text { const } .
$$

As already announced, this is exactly the asymptotic form of the numerical BB black strings $[1,2]$. 


\subsection{Superalgebras and Killing spinors}

There is another simple reason why the reduction and BPS properties had to work out correctly. Both the BB and CK solutions are 1/4 BPS, preserving 2 supercharges, with a susy enhancement near the horizon to 4 supercharges. Furthermore, in both cases Killing spinors obey

$$
\epsilon=\gamma_{x y} \epsilon
$$

where the $x, y$-directions denote the internal space $\Sigma$, on which the Killing spinor is constant. The full symmetry algebra on the 5 d horizon is [36]

$$
5 d: \mathrm{SU}(1,1 \mid 1) \times \mathrm{SU}(1,1) \times G_{\Sigma},
$$

where the symmetry group of $\Sigma$ can be $\mathrm{SU}(2), \mathrm{U}(1)^{2}$ for a sphere and torus or only a discrete group for a higher genus Riemann surface. The $4 \mathrm{~d}$ horizon has the symmetry algebra [37]

$$
4 d: \mathrm{SU}(1,1 \mid 1) \times G_{\Sigma},
$$

i.e. we have the exact same fermionic symmetries, but the extra bosonic $\mathrm{SU}(1,1)$ has disappeared, since $\mathrm{AdS}_{3}$ has an $\mathrm{SU}(1,1)^{2}$ isometries versus a single $\mathrm{SU}(1,1)$ for $\mathrm{AdS}_{2}$. Here lies the main reason why the reduction preserves the same amount of supersymmetry - we reduced along an isometry not only of the bosonic solution, but also of the Killing spinor. This is not guaranteed to be the always the case, see e.g. [38, 39], and it ensured the succes of the dimensional reduction. It is crucial to find the correct reduction ansatz, but the fact that we reduced over a full bosonic and fermionic isometry meant that there existed such an ansatz preserving all the supercharges of the original solution.

\subsection{Dual field theory and Cardy formula}

Following the above reduction on the boundary, one can find the corresponding CFT dual on the $4 \mathrm{~d}$ horizon. The theory dual to the horizon $\mathrm{AdS}_{3}$ of the BB solutions is a $(2,0)$ superconformal field theory in $2 \mathrm{~d}$ that can be derived from the compactification of twisted $N=4 \mathrm{SYM}$ on the corresponding Riemann surface $\Sigma$. The $(2,0)$ theory naturally lives on $\mathbb{R} \times \mathrm{S}^{1}$ and upon its reduction on the spatial circle becomes a superconformal quantum mechanics. We already know the central charge of the $(2,0)$ theory, which is the Brown-Henneaux central charge

$$
c=\frac{3 R_{A d S_{3}}}{2 G_{3}} .
$$

This result was derived independently on the dual side via c-extremization. Upon putting this CFT on a circle with momentum, cf. [40],

$$
L_{0}-\frac{c}{24}=\rho_{+}^{2} \frac{R_{A d S_{3}}}{4 G_{3}}=\rho_{+} \frac{\eta_{\Sigma} \tilde{R}_{\Sigma}^{2}}{2 \pi G_{4}},
$$

we see that the Cardy formula

$$
S_{\text {Cardy }}=2 \pi \sqrt{\frac{c}{6}\left(L_{0}-\frac{c}{24}\right)}=\frac{\eta_{\Sigma}}{G_{4}} \sqrt{\rho_{+} R_{A d S_{3}} R_{\Sigma}^{2} \tilde{R}_{\Sigma}^{2}}=\frac{\eta_{\Sigma} \tilde{R}_{\Sigma}^{2}}{G_{4}},
$$


reproduces exactly ${ }^{7}$ the macroscopic Bekenstein-Hawking entropy of the CK solution that is also the entropy of the BTZ black hole,

$$
S_{B H}=\frac{A_{\Sigma}}{4 G_{4}}=\frac{\eta_{\Sigma} \tilde{R}_{\Sigma}^{2}}{G_{4}}=\frac{2 \pi \eta_{\Sigma} \rho_{+} R_{A d S_{3}} R_{\Sigma}^{2}}{G_{5}}=\frac{\pi \rho_{+} R_{A d S_{3}}}{2 G_{3}}=\frac{A_{B T Z}}{4 G_{3}}=S_{B T Z} .
$$

This is a seemingly trivial AdS/CFT check, but it is important to stress that in this case we explicitly know the dual field theory and therefore have a true microscopic description of the black hole degrees of freedom. The nontrivial part of this derivation actually consists in finding the correct value of the central charge (3.34) directly from the field theory as done in $[1,2]$ via c-extremization. We stress again that we have essentially embedded a class of CK solutions inside type IIB supergravity via their relation with the BB solutions, such that now they have the interpretation of wrapped D3 branes. Thus it is not a surprise to see that the entropy scales with $N^{2}$ instead of $N^{3 / 2}$ (hidden in the scaling of the Newton constant $G_{3} \sim G_{4} \sim G_{5} \sim N^{-2}$ ). It also follows that the euclideanized version of the near-horizon geometry in section 3.2 is the gravity dual to be compared to field theory localization calculations of twisted $N=4 \mathrm{SYM}$ on $\mathrm{T}^{2} \times \Sigma^{2}$ (see [41] for some results in this direction).

\subsection{Extensions with extra charges}

It is interesting to observe that one could write more general CK solutions within the prepotential and FI terms considered in section 3.2, namely solutions with nonvanishing axions and more electric charges [33-35]. These have no known higher dimensional origin, but following the reduction rules in section 3.3 one can retrace what nonvanishing Re $z^{I}$ terms correspond to in the higher dimensional solution. One can add extra gauge fields

$$
A_{\gamma}^{I}=w^{I}
$$

which are essentially Wilson lines around the circle in $\mathrm{AdS}_{3}$. Upon reduction they produce non-vanishing axions and corresponding electric charges. It is interesting to observe that such Wilson lines already give nonvanishing electric charges in $5 \mathrm{~d}$ due to the Chern-Simons term in the supergravity lagrangian (3.1) and the fact that we have already switched on magnetic charges. This corresponds to a generalization of the BB solutions with electric charges and will be described more carefully in [42].

\section{$46 \mathrm{~d}$ to $5 \mathrm{~d}$ : black string $\rightarrow$ black hole/string}

The detailed example in the previous section illustrated the general principles of dimensional reduction in gauged supergravity. Other analogous examples can be explored across different dimensions, as long as there exists a suitable spatial isometry for dimensional reduction. In gauged supergravities such suitable spacetimes with $\mathrm{AdS}_{3}, \mathrm{~S}^{3}$ or other circle fibrations often arise as near-horizon geometries of various black branes. As discussed above, depending on the explicit Killing spinors of the original solution we can see if the dimensionally reduced solution also preserves supersymmetry or not.

\footnotetext{
${ }^{7}$ In the above derivation we used that the area of a unit Riemann surface $\Sigma$ is $4 \eta_{\Sigma}$ and depends on its genus as in $(3.6)$ of $[1,2]$.
} 
Here we sketch more briefly another example in 6d supergravity. The near-horizon geometry of BPS black strings found in [43] is $\mathrm{AdS}_{3} \times \mathrm{S}^{3}$. This is a solution of $N=$ $(1,0) \mathrm{SU}(2)$ gauged supergravity in $6 \mathrm{~d}$ with a nonvanishing $\mathrm{SU}(2)$ field strength $F^{I}$. The supergravity theory includes the gravity and tensor multiplets that include the metric, a scalar $\phi$ and tensor field strength $G$, and the above mentioned $\mathrm{SU}(2)$ gauge multiplet. The supersymmetric $\mathrm{AdS}_{3} \times \mathrm{S}^{3}$ background is given by:

$$
\begin{aligned}
\mathrm{d} s^{2} & =R_{1}^{2} \mathrm{~d} s_{A d S_{3}}^{2}+R_{2}^{2} \mathrm{~d} s_{S^{3}}^{2}, \\
G & =\frac{2\left(1-a^{2}\right)}{g^{2} R_{1} R_{2}^{2}}\left(\operatorname{Vol}_{R_{1}}\left(A d S_{3}\right)+\operatorname{Vol}_{R_{2}}\left(S^{3}\right)\right), \\
F^{I} & =\frac{\left(1-a^{2}\right)}{g} \epsilon^{I J K} e^{J K}, \quad e^{\sqrt{2} \phi}=\frac{g^{2} R_{2}^{2}}{2\left(1-a^{2}\right)} .
\end{aligned}
$$

In the above formulae $g$ is the gauge coupling constant, $a \equiv R_{2} / R_{1}, \epsilon^{I J K}$ are the $\mathrm{SU}(2)$ structure constants and $e^{I J}$ are two forms made from the vielbein on the three-sphere (see e.g. [36]). The supersymmetry is preserved due to the fact that the gauge field "cancels" the spin connection on the internal space. The solution is quarter-BPS, i.e. there are four conserved supercharges with a symmetry group

$$
\mathrm{SU}(1,1 \mid 1) \times \mathrm{SU}(1,1) \times \mathrm{SU}(2)^{2} .
$$

There is a clear similarity to the 5-dimensional case, and the fact that the Killing spinor transforms only under one $\mathrm{SU}(1,1)$ of the $\mathrm{AdS}_{3}$ isometry group and is scalar under rotations on the sphere means that now we have two ways of reducing along a circle while still preserving supersymmetry. One can reduce on a circle either inside $\mathrm{AdS}_{3}$ or inside $\mathrm{S}^{3}$. This leads to two different BPS solutions - $\mathrm{AdS}_{2} \times \mathrm{S}^{3}$ with $\mathrm{SU}(2)$ gauge fields or $\mathrm{AdS}_{3} \times \mathrm{S}^{2}$ with $\mathrm{U}(1)$ gauge fields.

The resulting $5 \mathrm{~d}$ theory coming from the reduction of the $N=(1,0)$ in $6 \mathrm{~d}$ has a $\mathrm{SU}(2) \times \mathrm{U}(1)$ gauging (the extra $\mathrm{U}(1)$ can be realized by Scherk-Scwarz reduction) and is of a runaway type, i.e. an $N=4$ theory with extra vectormultiplets and no extrema of the scalar potential, similar to some of the theories discussed in [36, 44, 45].

\subsection{Reduction along $\mathrm{AdS}_{3}$}

We can again use the BTZ form of the metric (3.11) and reduce the above solution along the circle. The resulting solution has the near-horizon geometry of a black hole in $5 \mathrm{~d}-$ $\mathrm{AdS}_{2} \times \mathrm{S}^{3}$ with the same $\mathrm{SU}(2)$ gauge field on the sphere. We further find two U(1) gauge fields carrying an electric charge - one coming from the $6 \mathrm{~d}$ tensor field and the other being the Kaluza-Klein vector. The fermionic symmetries remain the same, but the symmetry algebra does not contain the extra $\mathrm{SU}(1,1)$ factor found in $6 \mathrm{~d}$. Such a solution cannot be connected to any $\mathrm{AdS}_{5}$ vacuum, but near the horizon resembles already known attractors in $5 \mathrm{~d}$ (see e.g. [26, 36] for the case of supersymmetric $\mathrm{AdS}_{2} \times \mathrm{H}^{3}$ with an $\mathrm{SU}(2)$ gauge field).

\subsection{Reduction along $\mathrm{S}^{3}$}

To reduce along $\mathrm{S}^{3}$, one can write down the metric of the sphere in the form

$$
\mathrm{d} s_{S^{3} / \mathbb{Z}_{k}}^{2}=\frac{R_{2}^{2}}{4}\left(\sin ^{2} \theta \mathrm{d} \phi^{2}+\mathrm{d} \theta^{2}+(k \mathrm{~d} \psi+\cos \theta \mathrm{d} \phi)^{2}\right),
$$


with a slight generalization to a allow for quotients with a discrete group $\mathbb{Z}_{k}$. The resulting solution is less surprising - it becomes the near-horizon geometry of a black string, $\operatorname{AdS}_{3} \times \mathrm{S}^{2}$, with magnetic charges through the sphere. The symmetry algebra contains one less $\mathrm{SU}(2)$ factor compared to the $6 \mathrm{~d}$ case. Again, it is found in a theory with no $\mathrm{AdS}_{5}$ vacuum, but near the horizon it resembles the $\mathrm{BB}$ solutions in the previous section.

\subsection{Reduction along both $\mathrm{AdS}_{3}$ and $\mathrm{S}^{3}$}

Finally note that one can consecutively reduce along both circles in order to get a $4 \mathrm{~d}$ supersymmetric $\mathrm{AdS}_{2} \times \mathrm{S}^{2}$ solution. In this case already the $5 \mathrm{~d}$ supergravity is of runaway type and this continues to hold in $4 \mathrm{~d}$. The remaining supersymmetry algebra of this solution is $\mathrm{SU}(1,1 \mid 1) \times \mathrm{SU}(2)$ and can also be found within the near-horizon geometries of CK black holes and their generalizations.

\section{Acknowledgments}

I would like to thank S. Katmadas, D. Klemm, E. Ó Colgáin, A. Rota, A. Tomasiello, A. Veliz-Osorio, and A. Zaffaroni for enlightening discussions. I am supported in part by INFN, by the MIUR-FIRB grant RBFR10QS5J "String Theory and Fundamental Interactions", and by the MIUR-PRIN contract 2009-KHZKRX.

Open Access. This article is distributed under the terms of the Creative Commons Attribution License (CC-BY 4.0), which permits any use, distribution and reproduction in any medium, provided the original author(s) and source are credited.

\section{References}

[1] F. Benini and N. Bobev, Exact two-dimensional superconformal R-symmetry and c-extremization, Phys. Rev. Lett. 110 (2013) 061601 [arXiv:1211.4030] [INSPIRE].

[2] F. Benini and N. Bobev, Two-dimensional SCFTs from wrapped branes and c-extremization, JHEP 06 (2013) 005 [arXiv: 1302.4451] [INSPIRE].

[3] S.L. Cacciatori and D. Klemm, Supersymmetric AdS 4 black holes and attractors, JHEP 01 (2010) 085 [arXiv:0911.4926] [INSPIRE].

[4] B. de Wit, F. Vanderseypen and A. Van Proeyen, Symmetry structure of special geometries, Nucl. Phys. B 400 (1993) 463 [hep-th/9210068] [inSPIRE].

[5] T. Kugo and K. Ohashi, Supergravity tensor calculus in $5 D$ from $6 D$, Prog. Theor. Phys. 104 (2000) 835 [hep-ph/0006231] [INSPIRE].

[6] L. Andrianopoli, S. Ferrara and M.A. Lledó, Scherk-Schwarz reduction of D $=5$ special and quaternionic geometry, Class. Quant. Grav. 21 (2004) 4677 [hep-th/0405164] [INSPIRE].

[7] L. Andrianopoli, M.A. Lledó and M. Trigiante, The Scherk-Schwarz mechanism as a flux compactification with internal torsion, JHEP 05 (2005) 051 [hep-th/0502083] [INSPIRE].

[8] D. Gaiotto, A. Strominger and X. Yin, New connections between $4 D$ and 5D black holes, JHEP 02 (2006) 024 [hep-th/0503217] [INSPIRE]. 
[9] D. Gaiotto, A. Strominger and X. Yin, $5 D$ black rings and $4 D$ black holes, JHEP 02 (2006) 023 [hep-th/0504126] [INSPIRE].

[10] K. Behrndt, G. Lopes Cardoso and S. Mahapatra, Exploring the relation between $4 D$ and $5 D$ BPS solutions, Nucl. Phys. B 732 (2006) 200 [hep-th/0506251] [INSPIRE].

[11] H. Looyestijn, E. Plauschinn and S. Vandoren, New potentials from Scherk-Schwarz reductions, JHEP 12 (2010) 016 [arXiv: 1008.4286] [INSPIRE].

[12] N. Banerjee, B. de Wit and S. Katmadas, The off-shell 4D/5D connection, JHEP 03 (2012) 061 [arXiv:1112.5371] [INSPIRE].

[13] A. Strominger and C. Vafa, Microscopic origin of the Bekenstein-Hawking entropy, Phys. Lett. B 379 (1996) 99 [hep-th/9601029] [INSPIRE].

[14] J.M. Maldacena, A. Strominger and E. Witten, Black hole entropy in M-theory, JHEP 12 (1997) 002 [hep-th/9711053] [INSPIRE].

[15] K. Hristov and A. Rota, $6 D-5 D-4 D$ reduction of BPS attractors in flat gauged supergravities, arXiv: 1410.5386 [INSPIRE].

[16] E. Lozano-Tellechea, P. Meessen and T. Ortín, On $D=4, D=5, D=6$ vacua with eight supercharges, Class. Quant. Grav. 19 (2002) 5921 [hep-th/0206200] [INSPIRE].

[17] J.M. Maldacena and C. Núñez, Supergravity description of field theories on curved manifolds and a no go theorem, Int. J. Mod. Phys. A 16 (2001) 822 [hep-th/0007018] [INSPIRE].

[18] J.P. Gauntlett, Branes, calibrations and supergravity, hep-th/0305074 [INSPIRE].

[19] I.R. Klebanov and J.M. Maldacena, Superconformal gauge theories and non-critical superstrings, Int. J. Mod. Phys. A 19 (2004) 5003 [hep-th/0409133] [INSPIRE].

[20] S. Barisch-Dick, G. Lopes Cardoso, M. Haack and S. Nampuri, Extremal black brane solutions in five-dimensional gauged supergravity, JHEP 02 (2013) 103 [arXiv:1211.0832] [INSPIRE].

[21] P. Karndumri and E.Ó Colgáin, Supergravity dual of c-extremization, Phys. Rev. D 87 (2013) 101902 [arXiv:1302.6532] [INSPIRE].

[22] P. Karndumri and E.Ó Colgáin, 3D supergravity from wrapped D3-branes, JHEP 10 (2013) 094 [arXiv: 1307.2086] [INSPIRE].

[23] D. Klemm and W.A. Sabra, Supersymmetry of black strings in $D=5$ gauged supergravities, Phys. Rev. D 62 (2000) 024003 [hep-th/0001131] [InSPIRE].

[24] S. Cucu, H. Lü and J.F. Vazquez-Poritz, A supersymmetric and smooth compactification of M-theory to AdS $S_{5}$, Phys. Lett. B 568 (2003) 261 [hep-th/0303211] [INSPIRE].

[25] S. Cucu, H. Lü and J.F. Vazquez-Poritz, Interpolating from $A d S_{D-2} \times S^{2}$ to $A d S_{D}$, Nucl. Phys. B 677 (2004) 181 [hep-th/0304022] [INSPIRE].

[26] M. Naka, Various wrapped branes from gauged supergravities, hep-th/0206141 [INSPIRE].

[27] J.P. Gauntlett, O.A.P. Mac Conamhna, T. Mateos and D. Waldram, New supersymmetric $A d S_{3}$ solutions, Phys. Rev. D 74 (2006) 106007 [hep-th/0608055] [INSPIRE].

[28] A. Almuhairi and J. Polchinski, Magnetic AdS $\times R^{2}$ : supersymmetry and stability, arXiv: 1108.1213 [INSPIRE].

[29] L. Andrianopoli et al., $N=2$ supergravity and $N=2$ super Yang-Mills theory on general scalar manifolds: symplectic covariance, gaugings and the momentum map,

J. Geom. Phys. 23 (1997) 111 [hep-th/9605032] [INSPIRE]. 
[30] G. Dall'Agata and A. Gnecchi, Flow equations and attractors for black holes in $N=2 \mathrm{U}(1)$ gauged supergravity, JHEP 03 (2011) 037 [arXiv: 1012.3756] [INSPIRE].

[31] K. Hristov and S. Vandoren, Static supersymmetric black holes in AdS $S_{4}$ with spherical symmetry, JHEP 04 (2011) 047 [arXiv: 1012.4314] [INSPIRE].

[32] N. Halmagyi, BPS black hole horizons in $N=2$ gauged supergravity, JHEP 02 (2014) 051 [arXiv: 1308.1439] [INSPIRE].

[33] N. Halmagyi and T. Vanel, AdS black holes from duality in gauged supergravity, JHEP 04 (2014) 130 [arXiv: 1312.5430] [INSPIRE].

[34] S. Katmadas, Static BPS black holes in U(1) gauged supergravity, JHEP 09 (2014) 027 [arXiv: 1405.4901] [INSPIRE].

[35] N. Halmagyi, Static BPS black holes in AdS 4 with general dyonic charges, arXiv:1408.2831 [INSPIRE].

[36] K. Hristov and A. Rota, Attractors, black objects and holographic RG flows in $5 D$ maximal gauged supergravities, JHEP 03 (2014) 057 [arXiv: 1312.3275] [INSPIRE].

[37] K. Hristov, C. Toldo and S. Vandoren, On BPS bounds in $D=4 N=2$ gauged supergravity, JHEP 12 (2011) 014 [arXiv:1110.2688] [INSPIRE].

[38] M.J. Duff, H. Lü and C.N. Pope, Supersymmetry without supersymmetry, Phys. Lett. B 409 (1997) 136 [hep-th/9704186] [INSPIRE].

[39] K. Goldstein and S. Katmadas, Almost BPS black holes, JHEP 05 (2009) 058 [arXiv:0812.4183] [INSPIRE].

[40] P. Kraus, Lectures on black holes and the $A d S_{3} / C F T_{2}$ correspondence, Lect. Notes Phys. 755 (2008) 193 [hep-th/0609074] [INSPIRE].

[41] C. Closset and I. Shamir, The $N=1$ chiral multiplet on $T^{2} \times S^{2}$ and supersymmetric localization, JHEP 03 (2014) 040 [arXiv:1311.2430] [INSPIRE].

[42] K. Hristov and S. Katmadas, Wilson lines for AdS $S_{5}$ black strings, arXiv:1411.2432 [INSPIRE].

[43] M. Cariglia and O.A.P. Mac Conamhna, The general form of supersymmetric solutions of $N=(1,0) \mathrm{U}(1)$ and $\mathrm{SU}(2)$ gauged supergravities in six-dimensions,

Class. Quant. Grav. 21 (2004) 3171 [hep-th/0402055] [INSPIRE].

[44] M. Günaydin, L.J. Romans and N.P. Warner, Compact and noncompact gauged supergravity theories in five-dimensions, Nucl. Phys. B 272 (1986) 598 [INSPIRE].

[45] L.J. Romans, Gauged $N=4$ supergravities in five-dimensions and their magnetovac backgrounds, Nucl. Phys. B 267 (1986) 433 [INSPIRE]. 